\title{
Self-Assembled Hybrid Nanocomposites for Multimodal Imaging-Guided Photothermal Therapy of Lymph Node Metastasis
}

Wu Cai ${ }^{a, b, *}$, Guohua Fan ${ }^{a, *}$, Hui Zhou ${ }^{c, *}$, Lei Chen ${ }^{c}$, Jianxian Ge ${ }^{c}$, Baoxing Huang

${ }^{c}$, Dandan Zhou ${ }^{c}$, Jianfeng Zeng ${ }^{c, *}$, Qingqing Miao ${ }^{c,}{ }^{*}$, Chunhong $H u^{b,{ }^{*}}$

a Department of Radiology, Second Affiliated Hospital of Soochow University, Suzhou 215004, China

${ }^{b}$ Department of Radiology, the First Affiliated Hospital of Soochow University, Institute of Medical Imaging, Soochow University, Suzhou 215006, China

${ }^{\mathrm{c}}$ Center for Molecular Imaging and Nuclear Medicine, State Key Laboratory of Radiation Medicine and Protection, School for Radiological and Interdisciplinary Sciences (RAD-X), Soochow University, Collaborative Innovation Center of Radiological Medicine of Jiangsu Higher Education Institutions, Suzhou 215123, China

\section{Corresponding Author}

*Jianfeng Zeng, Email: jfzeng@suda.edu.cn

*Qingqing Miao, Email: qqmiao@suda.edu.cn

${ }^{*}$ Chunhong Hu, Email: hch5305@163.com 


\section{Table of Contents}

\section{Experimental sections}

2. Supporting Figures and Tables

\section{References}

\section{Experimental Section}

Materials. All chemical reagents were obtained from Sigma-Aldrich and used without further purification unless otherwise specified. Oleic acid-capped iron oxide nanoparticles $\left(\mathrm{Fe}_{3} \mathrm{O}_{4}\right)$ were provided by Xinying Bio-Medical Tech. Co, Ltd (Suzhou, China). Poly(lactic-co-glycolic acid) (PLGA) was from Jinnan Daigang Biological Material Co. Ltd. (Shangdong, China). D-luciferin, sodium salt and Calcein AM/PI double stain kit were purchased from Shanghai Yeasen Biotech Co. Ltd. (Shanghai, China). Dulbecco's modified eagle medium (DMEM) with L-glutamine and fetal bovine serum (FBS) were purchased from Sangon Biotech Co. Ltd. (Shanghai, China). Trypsin-EDTA $(0.05 \%)$ and penicillin-streptomycin were acquired from Life Technologies (India) Pvt Ltd. Hematoxylin and eosin (H\&E) staining was purchased from Kit Beijing Solarbio Science \& Technology Co., Ltd.. Ultrapure water was taken from Milli-Q Plus System (Millipore Corporation, Breford, USA).

Characterization. UV-Vis absorption spectra were recorded on a PerkinElmer LAMBDA 750 spectrophotometer. Fluorescence spectra were taken on a FLS 980 steady/transient state fluorescence spectrometer (Edinburgh Instruments). Transmission electron microscopy (TEM) was collected using FEI Tecnai G2 microscopes. Dynamic light scattering (DLS) measurements were recorded on a particle size analyzer (Nano ZS90, Malvern) at room temperature. Laser irradiation was carried out by an $808 \mathrm{~nm}$ NIR laser (Hi-Tech Optoelectronics Co. Ltd. Beijing, China). The IR images were recorded and quantified by a SC300 infrared camera (FLIR). Confocal fluorescence imaging was performed with a confocal microscopy (FV1200, Olympus). In vivo NIR-II fluorescence imaging was carried out using an IVIS Spectrum imaging system (PerkinElmer). 
Cell culture. The 4T1-luc and 4T1 murine breast cancer cells from American Type Culture Collection (ATCC) were cultured in DMEM containing 10\% FBS and 1\% penicillin/streptomycin antibiotics at $37{ }^{\circ} \mathrm{C}$ in $5 \% \mathrm{CO}_{2}$ and $95 \%$ humidified air, with medium changing every other day.

Cell imaging. To distinguish living and dead cells, a live/dead staining kit was applied accordingly. $3 \times 10^{5} 4 \mathrm{~T} 1$ cells were seeded into plates containing medium with or without $24 \mu \mathrm{g} \mathrm{mL} \mathrm{m}^{-1}$ FIP for $24 \mathrm{~h}$. After incubation and washing with PBS, the $4 \mathrm{~T} 1$ cells were irradiated with or without $808 \mathrm{~nm}$ laser at $0.75 \mathrm{~W} \mathrm{~cm}^{-2}$ power density for 10 min, followed by staining with Calcein AM $(2 \mu \mathrm{M})$ and PI $(4.5 \mu \mathrm{M})$ for 30 min. Fluorescence of Calcein AM and PI were gathered at a green channel $(515 \mathrm{~nm}$, excitation wavelength: $490 \mathrm{~nm})$ and red channel (617 nm, excitation wavelength: 535 $\mathrm{nm})$, respectively.

Tumor mouse model. Specific pathogen free (SPF) grade Balb/c female mice were purchased from Shanghai SLAC Laboratory Animal Co., Ltd. and used under protocols approved by the Soochow University Laboratory Animal Center. $1 \times 10^{6}$ 4T1-luc murine breast cancer cells suspended in $40 \mu \mathrm{L}$ PBS were injected subcutaneously on the left footpad. Metastatic tumors were allowed to grow to spherical hard lumps in their inner knee (approximately 7 days) before imaging and PTT experiments.

\section{Multimodal imaging.}

NIR-II fluorescence imaging: NIR-II fluorescence imaging was examined with a NIR-II Imaging System (Serious II 900-1700) manufactured by Suzhou NIR-Optics Co. Ltd. (Suzhou, China). Mice were firstly anesthetized with 3\% isoflurane and oxygen gas (flow rate: $\left.0.5 \mathrm{~L} \mathrm{~min}^{-1}\right)$. Subsequently, FIP $\left(50 \mu \mathrm{L}, 120 \mu \mathrm{g} \mathrm{mL}^{-1}\right)$ were injected into the primary tumor resided in the footpad followed by the acquisition of NIR-II fluorescence images with $808 \mathrm{~nm}$ laser at $177 \mathrm{~mW} \mathrm{~cm}^{-2}$. All NIR-II fluorescence images were captured with a $1000 \mathrm{~nm}$ long-pass filter and an exposure time of $200 \mathrm{~ms}$.

PAI: PAI was performed with a Multispectral Optoacoustic Tomography scanner 
(MSOT, iThera Medical GmbH, inSight/inVision 256). In vitro PA images of FIP at concentrations of $0,3,6,12$, and $24 \mu \mathrm{g} \mathrm{mL}^{-1}$ were acquired at $750 \mathrm{~nm}$. For in vivo PA imaging, the 4T1 tumor-bearing mice were anesthetized with isoflurane, and placed into a water bath to maintain their body temperature at $37^{\circ} \mathrm{C}$. FIP were intratumoral injected into the mice, and images were captured at $0,1,2,3$, and $6 \mathrm{~h}$ post-injection.

MRI: The MR images were acquired on a 3.0 T clinical MRI instrument (Achieva 3.0 T TX, Philips). For in vitro MRI, FIP at different concentrations $(0,0.05,0.1,0.2,0.5$ $\mathrm{mM}$ ) of Fe ion were scanned under a MRI scanner with $\mathrm{T}_{2}$ weighted imaging at room temperature. Then the signal intensity was measured within a manually drawn region of interest (ROT) for each sample. Furthermore, relaxation rates $R_{2}\left(R_{2}=1 / \mathrm{T}_{2}\right)$ were calculated from $\mathrm{T}_{2}$ values at different concentrations of Fe. For in vivo MR imaging, mice were abdominally anesthetized and then were intratumorally injected with FIP (120 $\left.\mu \mathrm{g} \mathrm{mL}^{-1}, 50 \mu \mathrm{L}\right) . \mathrm{T}_{2}$ weighted images were captured using FSE sequence at designed time points. $T_{2}$ weighted imaging was scanned with the following parameters: $\mathrm{TR}=2000 \mathrm{~ms}$; $\mathrm{TE}=106.4 \mathrm{~ms}$; slice thickness $=2.0 \mathrm{~mm}$; slice spacing $=$ $0.2 \mathrm{~mm} ; \mathrm{FOV}=10 \times 10 \mathrm{~cm}^{2}$; matrix size $=224 \times 192$. Then the signal intensity of metastatic lymph nodes in their inner knee was measured within a manually drawn ROI for each mouse.

SPECT imaging: SPECT images were obtained with a U-SPECT+/CT from MILabs (Hong Kong Maylin International Trade Co., Ltd). Firstly, pre-contrast images of mice were captured. After intratumoral injection of FIP- ${ }^{99 m} \mathrm{Tc}\left(120 \mu \mathrm{g} \mathrm{mL}^{-1}, 50 \mu \mathrm{L}\right.$, $20 \mu \mathrm{Ci} 99 \mathrm{~m} \mathrm{Tc})$, SPECT/CT images were acquired at different time points post-injection.

Examination of lung metastasis. To confirm whether lung metastasis occurred or not, mice were sacrificed at $\mathrm{t}=45$ days post-injection of FIP. The isolated gross specimen of lungs were scrutinized to detect abnormal metastatic lesions. Then the lungs and lymph nodes were soaked in $10 \mathrm{~mL}$ formalin solutions for 2-3 days at room temperature. For pathologic study, the lung tissue sections of different thicknesses were processed with $10 \%$ formalin to make into paraffin, which was further cut into 
8-micrometer thick slices and treated with H\&E staining.

\section{Supporting Figures and Tables}
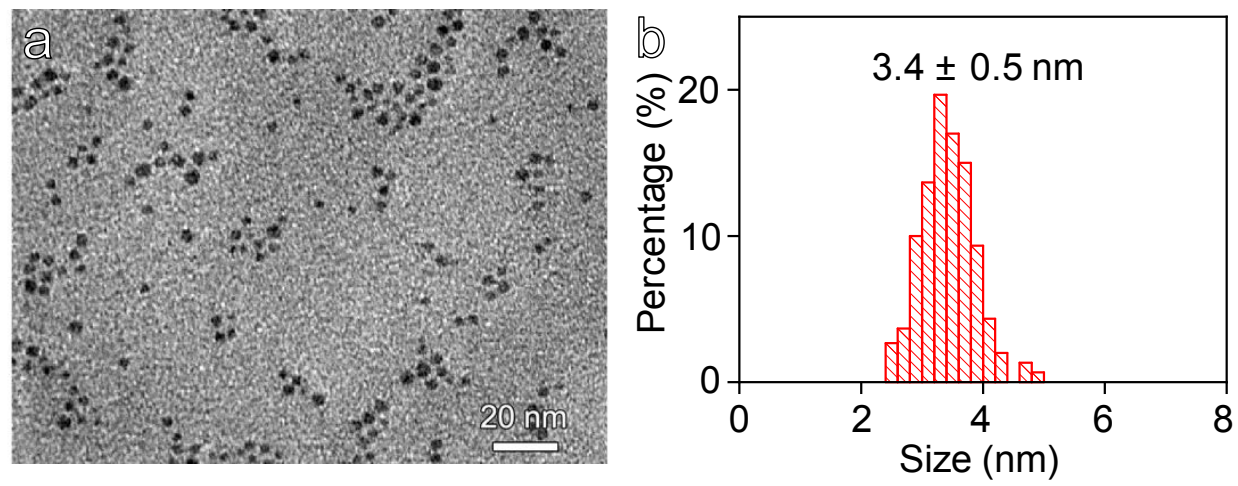

Figure S1. Representative TEM images of $\mathrm{Fe}_{3} \mathrm{O}_{4}$ (a) and its size statistics (b).

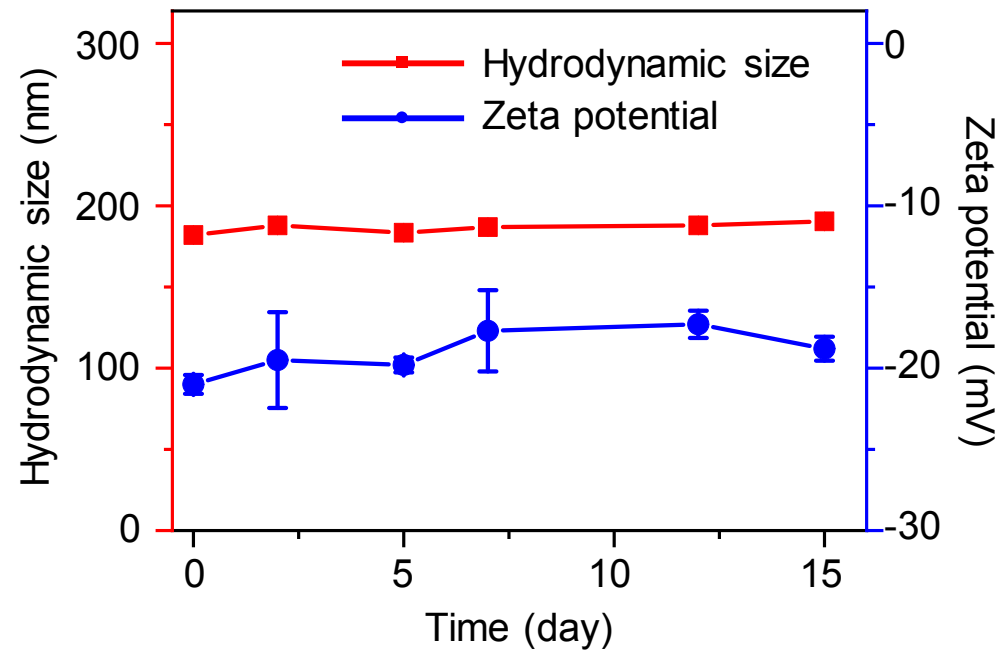

Figure S2. Stability study of FIP in FBS after storage over a period of 15 days. 


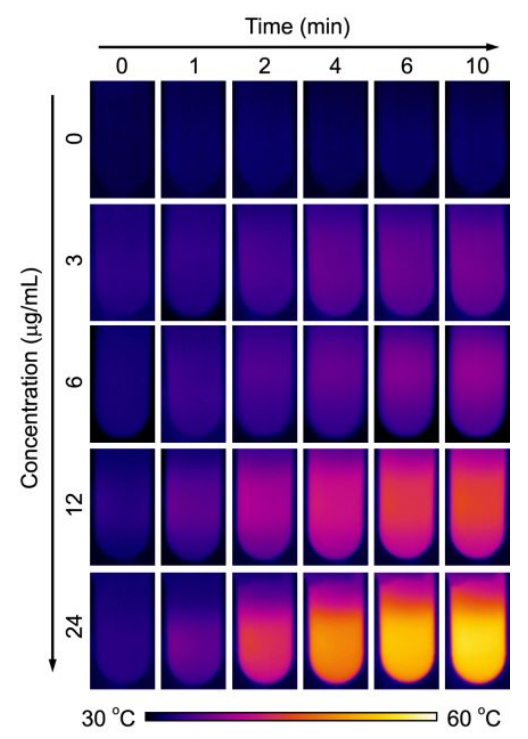

Figure S3. Infrared thermal images of FIP solutions at various concentrations as a function of laser irradiation time (NIR laser wavelength: $808 \mathrm{~nm}$; laser power: $0.75 \mathrm{~W}$ $\left.\mathrm{cm}^{-2}\right)$.
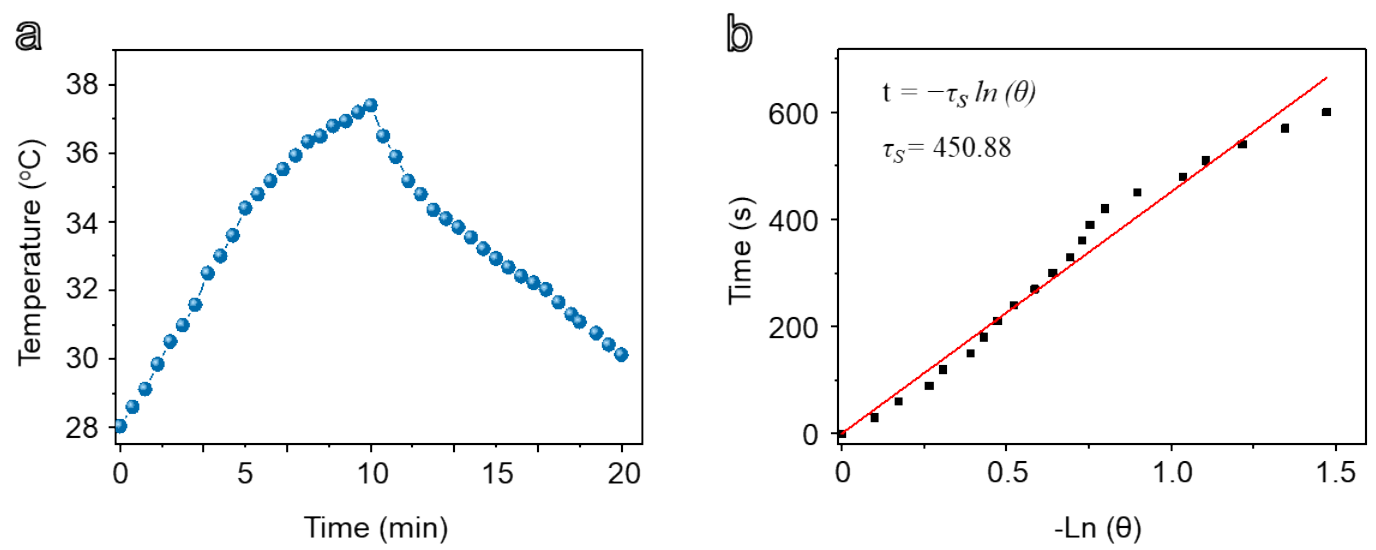

Figure S4. (a) Photothermal effect of FIP solutions $\left(12 \mu \mathrm{g} \mathrm{mL}^{-1}\right)$ after irradiation with the $808 \mathrm{~nm}$ NIR laser $\left(0.75 \mathrm{~W} \mathrm{~cm}^{-2}\right)$. The irradiation time lasted for $10 \mathrm{~min}$, and then the laser was turned off. (b) Plot of cooling time versus negative natural logarithm of the temperature driving force. 


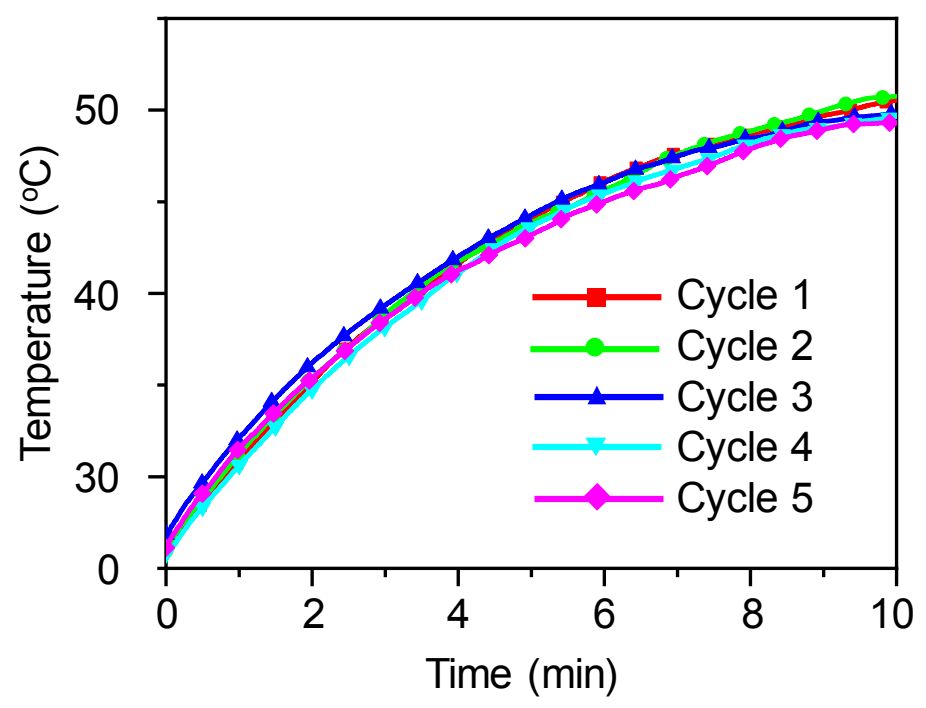

Figure S5. The temperature of FIP solutions as a function of laser irradiation time under five consecutive heating-cooling cycles.
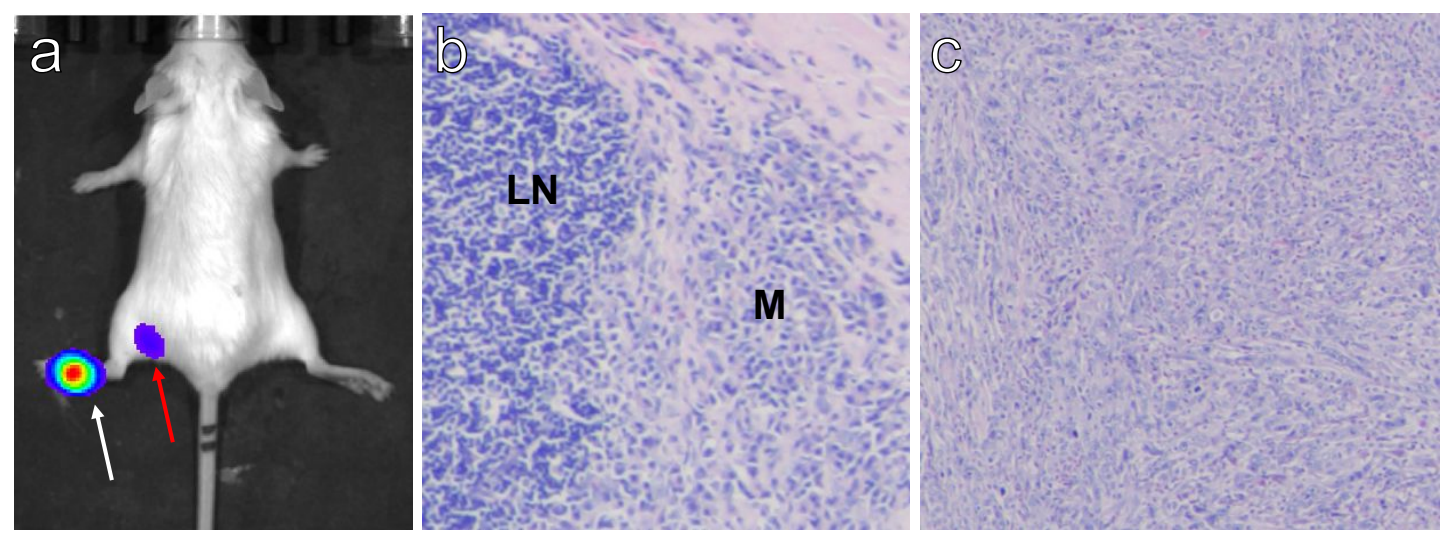

Figure S6. The lymphatic metastasis model. (a) Bioluminescence imaging of primary 4T1 tumor (white arrow) with lymph node metastasis (red arrow). (b) H\&E staining of left popliteal lymph node (LN: lymph node, M: metastasis). (c) H\&E staining of footpad tumor. 


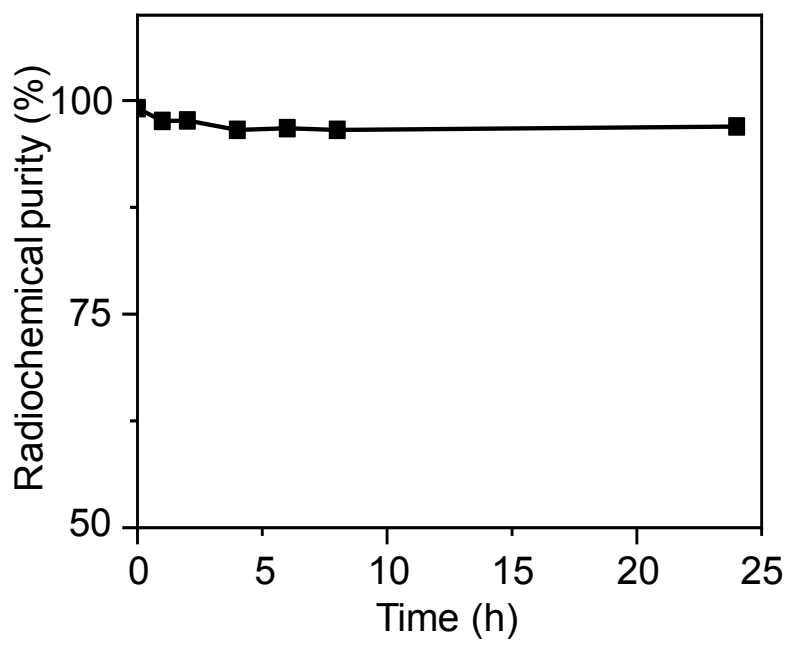

Figure S7. The radiochemical purity of FIP- ${ }^{99 \mathrm{~m} T c}$ solution over a period of $24 \mathrm{~h}$.

Table S1. Summary of PTT performance of therapeutic agents $(\Delta \mathrm{T}=$ temperature increase; $\eta=$ photothermal conversion efficiency).

\begin{tabular}{|c|c|c|c|}
\hline Agents & Laser irradiation & PTT performance & Ref. \\
\hline Bi-LyP-1 NPs & $\begin{array}{c}1064 \mathrm{~nm}\left(0.6 \mathrm{~W} \mathrm{~cm}^{-2}, 20\right. \\
\mathrm{min})\end{array}$ & $\Delta \mathrm{T} \approx 15^{\circ} \mathrm{C}(0.6 \mathrm{mg}), \eta=32.2 \%$ & 1 \\
\hline $\mathrm{Fe}_{3} \mathrm{O}_{4} @ \mathrm{CuS}$ & $1064 \mathrm{~nm}\left(3 \mathrm{~W} \mathrm{~cm}^{-2}, 15 \mathrm{~min}\right)$ & $\Delta \mathrm{T} \approx 24{ }^{\circ} \mathrm{C}(300 \mathrm{ppm} \mathrm{Cu}$, in vitro $), \eta=19.2 \%$ & 2 \\
\hline $\mathrm{Bi}_{2} \mathrm{~S}_{3}$ & $808 \mathrm{~nm}\left(1 \mathrm{~W} \mathrm{~cm}^{-2}, 10 \mathrm{~min}\right)$ & $\Delta \mathrm{T} \approx 50{ }^{\circ} \mathrm{C}\left(1 \mathrm{mg} \mathrm{mL}^{-1}\right), \eta=28.1 \%$ & 3 \\
\hline ICG & $808 \mathrm{~nm}\left(0.5 \mathrm{~W} \mathrm{~cm}^{-2}, 5 \mathrm{~min}\right)$ & $\Delta \mathrm{T} \approx 45.4^{\circ} \mathrm{C}\left(20 \times 10^{-6} \mathrm{M}\right), \eta=36.6 \pm 2.1 \%$ & 4 \\
\hline $\mathrm{Bi}_{2} \mathrm{~S}_{3}$ & $\begin{array}{c}808 \mathrm{~nm}\left(0.75 \mathrm{~W} \mathrm{~cm}^{-2}, 10\right. \\
\mathrm{min})\end{array}$ & $\Delta \mathrm{T} \approx 21.5^{\circ} \mathrm{C}\left(120 \mu \mathrm{g} \mathrm{mL}^{-1}\right), \eta=51 \%$ & 5 \\
\hline $\mathrm{Cu}_{2-\mathrm{x}} \mathrm{Se}$ & $\begin{array}{c}808 \mathrm{~nm}\left(0.75 \mathrm{~W} \mathrm{~cm}^{-2}, 10\right. \\
\mathrm{min})\end{array}$ & $\Delta \mathrm{T} \approx 18.5^{\circ} \mathrm{C}\left(20 \mu \mathrm{g} \mathrm{mL}^{-1}\right), \eta=64.8 \%$ & 6 \\
\hline DPP-BT & $730 \mathrm{~nm}\left(1 \mathrm{~W} \mathrm{~cm}^{-2}, 10 \mathrm{~min}\right)$ & $\Delta \mathrm{T} \approx 45^{\circ} \mathrm{C}(26.4 \mu \mathrm{g}), \eta=50.0 \%$ & 7 \\
\hline NLG919/PCB & $808 \mathrm{~nm}\left(0.3 \mathrm{~W} \mathrm{~cm}^{-2}, 6 \mathrm{~min}\right)$ & $\Delta \mathrm{T} \approx 57^{\circ} \mathrm{C}\left(40 \mu \mathrm{g} \mathrm{mL}^{-1} \mathrm{PCB}\right), \eta=42.2 \%$ & 8 \\
\hline $\mathrm{Fe} @ \mathrm{Fe}_{3} \mathrm{O}_{4}$ & $\begin{array}{c}808 \mathrm{~nm}\left(0.38 \mathrm{~W} \mathrm{~cm}^{-2}, 10\right. \\
\mathrm{min})\end{array}$ & $\Delta \mathrm{T} \approx 12.5^{\circ} \mathrm{C}\left(50 \mu \mathrm{g} \mathrm{mL}^{-1}\right), \eta=20.3 \%$ & 9 \\
\hline $\mathrm{Gd} / \mathrm{CuS}$ & $808 \mathrm{~nm}\left(0.8 \mathrm{~W} \mathrm{~cm}^{-2}, 5 \mathrm{~min}\right)$ & $\Delta \mathrm{T} \approx 38.7^{\circ} \mathrm{C}\left(50 \mu \mathrm{g} \mathrm{mL}^{-1}\right), \eta=70.1 \%$ & 10 \\
\hline
\end{tabular}




\begin{tabular}{|l|c|c|c|}
\hline $\mathrm{Fe}^{3+} / \mathrm{GA}$ & $808 \mathrm{~nm}\left(0.5 \mathrm{~W} \mathrm{~cm}^{-2}, 10 \mathrm{~min}\right)$ & $\Delta \mathrm{T} \approx 28.3{ }^{\circ} \mathrm{C}(22.5 \mu \mathrm{g} \mathrm{mL}-1 \mathrm{Fe}), \eta=67.4 \pm 5.1 \%$ & 11 \\
\hline $\mathrm{IR} 1048$ & $980 \mathrm{~nm}(0.1 \mathrm{~W} \mathrm{~cm}-2,2 \mathrm{~min})$ & $\Delta \mathrm{T} \approx 27 .{ }^{\circ} \mathrm{C}, \eta=20.2 \%$ & 12 \\
\hline TQTPA & $808 \mathrm{~nm}(1.5 \mathrm{~W} \mathrm{~cm}-10 \mathrm{~min})$ & $\Delta \mathrm{T} \approx 40.5^{\circ} \mathrm{C}(300 \mu \mathrm{g} \mathrm{mL}-1), \eta=22.0 \%$ & 13 \\
\hline
\end{tabular}

\section{References}

(1) Yu, X. J.; Li, A.; Zhao, C. Z.; Yang, K.; Chen, X. Y.; Li, W. W. Ultrasmall Semimetal Nanoparticles of Bismuth for Dual-Modal Computed Tomography/Photoacoustic Imaging and Synergistic Thermoradiotherapy. ACS Nano 2017, 11, 3990-4001.

(2) Wu, Z. C.; Li, W. P.; Luo, C. H.; Su, C. H. o.; Yeh, C. S. Rattle-Type $\mathrm{Fe}_{3} \mathrm{O}_{4} @$ CuS Developed to Conduct Magnetically Guided Photoinduced Hyperthermia at First and Second NIR Biological Windows. Adv. Funct. Mater. 2015, 25, 6527-6537.

(3) Liu, J.; Zheng, X. P.; Yan, L.; Zhou, L. J.; Tian, G.; Yin, W. Y.; Wang, L. M.; Liu, Y.; Hu, Z. B.; Gu, Z. J.; Chen, C. Y.; Zhao, Y. L. Bismuth Sulfide Nanorods as a Precision Nanomedicine for in Vivo Multimodal Imaging-Guided Photothermal Therapy of Tumor. ACS Nano 2015, 9, 696-707.

(4) Du, W.; Chong, Y. Y.; Hu, X. M.; Wang, Y. F.; Zhu, Y.; Chen, J. H.; Li, X. X.; Zhang, Q.; Wang, G. F.; Jiang, J.; Liang, G. L. Increasing Photothermal Efficacy by Simultaneous Intra- and Intermolecular Fluorescence Quenching. Adv. Funct. Mater. 2019, 30, 1908073.

(5) Wang, Y.; Wu, Y. Y.; Liu, Y. J.; Shen, J.; Lv, L.; Li, L. B.; Yang, L. C.; Zeng, J. F.; Wang, Y. Y.; Zhang, L. W.; Li, Z.; Gao, M. Y.; Chai, Z. F. BSA-Mediated Synthesis of Bismuth Sulfide Nanotheranostic Agents for Tumor Multimodal Imaging and Thermoradiotherapy. Adv. Funct. Mater. 2016, 26, 5335-5344.

(6) Zhang, S.; Sun, C.; Zeng, J.; Sun, Q.; Wang, G.; Wang, Y.; Wu, Y.; Dou, S.; Gao, M.; Li, Z. Ambient Aqueous Synthesis of Ultrasmall PEGylated $\mathrm{Cu}_{2-\mathrm{x}} \mathrm{Se}$ Nanoparticles as a Multifunctional Theranostic Agent for Multimodal Imaging Guided Photothermal Therapy of Cancer. Adv. Mater. 2016, 28, 8927-8936.

(7) Wang, Q.; Dai, Y. N.; Xu, J. Z.; Cai, J.; Niu, X. R.; Zhang, L.; Chen, R. F.; Shen, Q. M.; Huang, W.; Fan, Q. L. All-in-One Phototheranostics: Single Laser Triggers NIR-II Fluorescence/Photoacoustic Imaging Guided Photothermal/Photodynamic/Chemo Combination Therapy. Adv. Funct. Mater. 2019, 29, 1901480.

(8) Li, J. C.; Cui, D.; Huang, J.; He, S. S.; Yang, Z.; Zhang, Y. B.; Luo, Y.; Pu, K. Y. Organic Semiconducting Pro-nanostimulants for Near-Infrared Photoactivatable Cancer Immunotherapy. Angew. Chem. Int. Ed. 2019, 58, 12680-12687; Angew.Chem. 2019, 131,12810-12817.

(9) Zhou, Z.; Sun, Y. N.; Shen, J. C.; Wei, J.; Yu, C.; Kong, B.; Liu, W.; Yang, H.; Yang, S. Q.; Wang, W. Iron/Iron Oxide Core/Shell Nanoparticles for Magnetic Targeting MRI and Near-Infrared Photothermal Therapy. Biomaterials 2014, 35, 7470-7478.

(10) Shi, H.; Sun, Y.; Yan, R. Q.; Liu, S.; Zhu, L.; Liu, S. L.; Feng, Y. Z.; Wang, P.; He, J.; Zhou, Z. Y.; Ye, D. J. Magnetic Semiconductor Gd-Doping CuS Nanoparticles as Activatable Nanoprobes for Bimodal Imaging and Targeted Photothermal Therapy of Gastric Tumors. Nano Lett. 2019, 19, 937-947.

(11) Chen, L.; Chen, J. Y.; Qiu, S. S.; Wen, L.; Wu, Y.; Hou, Y.; Wang, Y.; Zeng, J. F.; Feng, Y.; Li, Z.; Shan, H.; Gao, M. Y. Biodegradable Nanoagents with Short Biological Half-Life for 
SPECT/PAI/MRI Multimodality Imaging and PTT Therapy of Tumors. Small 2018, 14, 1702700.

(12) Ge, X. G.; Fu, Q. R.; Su, L. C.; Li, Z.; Zhang, W. M.; Chen, T.; Yang, H. H.; Song, J. B. Light-Activated Gold Nanorod Vesicles With NIR-II Fluorescence and Photoacoustic Imaging Performances for Cancer Theranostics. Theranostics 2020, 10, 4809-4821.

(13) Wang, Y.; Zhang, W.; Sun, P.; Cai, Y.; Xu, W.; Fan, Q.; Hu, Q.; Han, W. A Novel Multimodal NIR-II Nanoprobe for the Detection of Metastatic Lymph Nodes and Targeting Chemo-Photothermal Therapy in Oral Squamous Cell Carcinoma. Theranostics 2019, 9, 391-404. 